\title{
Focusing Flexural Waves in Beams for Precisely Controlled Dynamic Fracture
}

\author{
Valentin van Gemmeren, ${ }^{*}$ Bernhard Zybach, and Jurg Dual \\ Institute for Mechanical Systems, ETH Zurich, 8092 Zurich, Switzerland
}

(Received 12 October 2017; revised manuscript received 24 May 2018; published 8 October 2018)

\begin{abstract}
We show that flexural waves can be focused in a beam to an extent that induces precisely controlled dynamic fracture of the beam. Flexural waves are excited at one end of a finite beam and focus at another location along the beam to form a shorter but much larger bending-moment pulse. The strong focusing is achieved by use of the frequency dependence of the phase and group velocity of flexural waves, as well as the superposition of multiple reflections. Amplification of the actuator input by a factor of 20 is achieved solely by wave focusing, proving the potential of such a technique.
\end{abstract}

DOI: 10.1103/PhysRevApplied.10.044021

\section{INTRODUCTION}

Some mechanical waves, such as flexural waves and Lamb waves, show dispersion, meaning that their phase and group velocities are frequency dependent [1]. This causes the distortion of propagating broadband pulses of such waves, and spreads out the energy that is carried by the pulse in space and time. While the distortion of a propagating wave package due to dispersion may be considered detrimental in some applications [2], it also opens opportunities in others. For instance, the amount of distortion a wavelet has undergone reveals information about the distance it has covered. This feature has been used in guided-wave-testing applications to locate cracks in beams [3,4] and plates [5] with use of only a single-point measurement of the acoustic emission. Similarly, cracks were identified in tubes with use of only circumferential measurements at one location along the tube [6].

The aforementioned methods rely on the time-reversal symmetry of acoustic waves to retrace the propagation path of the measured waves. The time-reversal symmetry has been studied extensively by Fink with both acoustic waves [7-10] and electromagnetic waves [11] and has been used to focus waves via randomly distributed scatterers [12] and metamaterials [13-15], with applications in medicine, undersea communications, and hydrodynamics [7].

Another application of mechanical waves can be found in material testing at high strain-rates with split Hopkinson (Kolsky) pressure bars [16,17]. Here, a propagating pressure-wave pulse is used to induce fracture of the

\footnotetext{
*vangemmeren@imes.mavt.ethz.ch
}

Published by the American Physical Society under the terms of the Creative Commons Attribution 4.0 International license. Further distribution of this work must maintain attribution to the author(s) and the published article's title, journal citation, and DOI. material sample under dynamic conditions. The pressurewave pulse is created by hitting the bar with a striker, and it can be shaped, for example, by use of a specially designed striker [18] or by insertion of tip material between the striker and the bar [19]. While the classic Kolsky bar setup is used for compression experiments, modifications for tensile [20,21] and torsional [22] experiments exist. Different experimental implementations for bending have been reported as well, but are rare [23-26]. They offer an alternative to bending tests using drop-weight towers, which struggle with a moving specimen [27]. Especially for brittle materials, such as glass, ceramics, or concrete, questions regarding the fracture mechanisms at high strain rates remain open, and appropriate experimental setups are being developed [28].

Here, we propose a method that uses time-reversal techniques and dispersion to create a precisely controlled, localized, high-power wave pulse in a glass beam to induce dynamic fracture. Exploiting the dispersive nature of flexural waves, which causes the separation of different wavelengths, allows an actuation time that is much longer than the duration of the focused pulse. A somewhat similar method is used in optics for pulse shaping [29]. The time-reversal invariance is used to compute the actuation corresponding to the desired pulse shape and focusing location.

A schematic of the energy-focusing procedure is shown in Fig. 1: An electromechanical transducer is glued to one end of a glass beam and is excited in such a way that the resulting bending waves focus at an arbitrarily chosen location along the beam. At this location, which we call the "focal point," the time history of the bending moment is that of a Ricker wavelet with a certain peak width (Fig. 2). To obtain the corresponding excitation signal, the defocusing of the bending-moment pulse is simulated by its being set as a boundary condition at the focal point (time $t_{S}$ running to the left in the time-space map). Taking the 


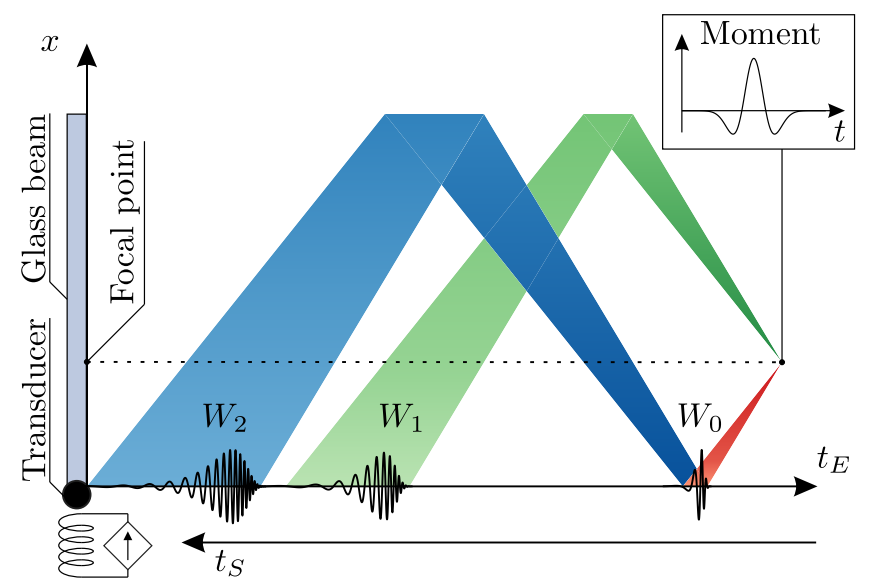

FIG. 1. Time-space map of the energy-focusing procedure. Wavelets are excited at one end of a glass beam with an electromechanical transducer. The propagation paths of the three wavelets $W_{0}, W_{1}$, and $W_{2}$ are shown exemplarily in a time-space map. After being excited, $W_{0}$ focuses to a narrow peak at the focal point, whereas $W_{1}$ and $W_{2}$ are reflected once and twice, respectively, before focusing at the same point in space and time. The excitation signal is obtained by simulation of the defocusing of the narrow peak (time $t_{S}$ running to the left) and subsequent reversal of the time ( $t_{E}$ running to the right).

simulated time history of the bending moment at the end of the beam and reversing the time to $t_{E}$ (running to the right) yields the appropriate excitation signal. This is done not only for one wavelet $W_{0}$ taking the direct path from the transducer to the focal point but also for wavelets $W_{r}$ undergoing $r=0,1, \ldots, n$ reflections at the ends of the beam before focusing at the focal point. Thus, the time in which the transducer is active and energy can be pumped into the beam is increased. Finally, the excitation signal for the transducer is composed of the weighted sum of all computed wavelets $W_{0}$ to $W_{n}$.

The excitation of a new wavelet $W_{r}$ coincides with the reflection of the previously created wavelets $W_{r+m}$, where $m$ is an even number, as illustrated in Fig. 1 for wavelets $W_{0}$ and $W_{2}$. The reflection of a previous wavelet, however, does not affect the excitation of a new one, as the deformations stay small and the system behaves linearly. The
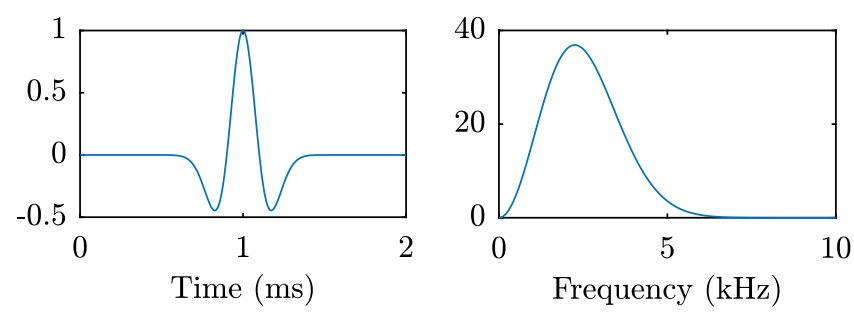

FIG. 2. Time history and frequency content of the focused pulse (Ricker wavelet). propagating part of the reflected wavelet and the propagating part of the newly created wavelet are identical in shape and phase, as they are designed to focus at the same point in space and time.

In Sec. II, we discuss the computation of the excitation signal for wave focusing. This comprises the modeling of the beam and the magnetic sphere using spectral elements, the simulation of the defocusing, some considerations regarding the compensation of the evanescent mode, and finally the optimal composition of the excitation signal from multiple wavelets. In Sec. III, the experimental setup is detailed, the characterization of the beam and transducer is explained, and the results are presented. Finally, the results and limitations of the method are discussed, and a conclusion is given.

\section{METHOD}

\section{A. Modeling of the beam}

To simulate the propagation of flexural waves, the glass beam is modeled according to Timoshenko beam theory [30]: The deformation is described by the lateral displacement $w(x, t)$ of the centerline and the angle of rotation $\phi(x, t)$ of the cross section. The governing equations are

$$
\begin{aligned}
G A K_{1} \frac{\partial}{\partial x}\left(\frac{\partial w}{\partial x}-\phi\right) & =\rho A \ddot{w}, \\
E I \frac{\partial^{2} \phi}{\partial x^{2}}+G A K_{1}\left(\frac{\partial w}{\partial x}-\phi\right) & =\rho I K_{2} \ddot{\phi},
\end{aligned}
$$

where $G$ is the shear modulus, $A$ the cross-sectional area, $\rho$ the density, $E$ the Young's modulus, and $I$ the second moment of inertia. $K_{1}$ and $K_{2}$ incorporate the effect of shear and are set to $\pi^{2} / 12$ and 1 , respectively [31]. Two wave modes with wave numbers $k_{1}$ and $k_{2}$ satisfy these equations and are referred to as the "flexural-wave mode" and "thickness-shear mode." In the relevant frequency domain, the first wave number $k_{1}$ is real (i.e., a propagating wave), whereas the second wave number $k_{2}$ is purely imaginary (i.e., an evanescent mode). In general, when the beam is actuated, both wave modes are excited. So, while only the propagating mode is useful for wave focusing, the evanescent mode has to be considered in the boundary conditions too.

The equations of motion are solved in the frequency domain with use of the spectral-element approach proposed by Doyle [31]. We follow Doyle's notation and use semi-infinite spectral elements to model the glass beam. For a semi-infinite beam extending from $x=0$ into the positive $x$-direction, the deformation is given by two dynamic shape functions $\hat{w}(x)$ and $\hat{\phi}(x)$, where the time dependence $e^{i \omega t}$ is suppressed and a superposed caret 
denotes complex amplitudes:

$$
\begin{aligned}
& \hat{w}(x)=R_{1} \hat{A} e^{-i k_{1} x}+R_{2} \hat{B} e^{-i k_{2} x} \\
& \hat{\phi}(x)=\hat{A} e^{-i k_{1} x}+\hat{B} e^{-i k_{2} x}
\end{aligned}
$$

where $\hat{A}$ and $\hat{B}$ are the frequency-dependent coefficients defining the amplitude and phase of the two wave modes. The two amplitude ratios $R_{j}$ are given by

$$
R_{j}=\frac{i k_{j} G A K_{1}}{G A K_{1} k_{j}^{2}-\rho A \omega^{2}} .
$$

The dynamic-stiffness matrix $\hat{\mathbf{K}}_{R}$, relating the nodal displacement $\hat{w}_{N}=\hat{w}(0)$ and rotation $\hat{\phi}_{N}=\hat{\phi}(0)$ to the nodal force $\hat{F}$ and moment $\hat{T}$, is

$$
\underbrace{E I\left(\begin{array}{ll}
\hat{k}_{11} & \hat{k}_{12} \\
\hat{k}_{21} & \hat{k}_{22}
\end{array}\right)}_{\hat{\mathbf{k}}_{R}} \underbrace{\left(\begin{array}{c}
\hat{w}_{N} \\
\hat{\phi}_{N}
\end{array}\right)}_{\hat{\boldsymbol{u}}_{N}}=\underbrace{\left(\begin{array}{c}
\hat{F} \\
\hat{T}
\end{array}\right)}_{\hat{\boldsymbol{F}}_{N}},
$$

where $\hat{k}_{11}=\left(k_{2}^{2}-k_{1}^{2}\right) /\left(R_{1}-R_{2}\right), \hat{k}_{12}=\hat{k}_{21}=i\left(k_{1}-k_{2}\right) /$ $\left(R_{1}-R_{2}\right)$, and $\hat{k}_{22}=i\left(R_{1} k_{2}-R_{2} k_{1}\right) /\left(R_{1}-R_{2}\right)$.

Similarly, the dynamic-stiffness matrix $\hat{\mathbf{K}}_{L}$ for a semiinfinite beam extending from $x=0$ into the negative $x$-direction is

$$
\underbrace{E I\left(\begin{array}{cc}
\hat{k}_{11} & -\hat{k}_{12} \\
-\hat{k}_{21} & \hat{k}_{22}
\end{array}\right)}_{\hat{\mathbf{k}}_{L}} \underbrace{\left(\begin{array}{c}
\hat{w}_{N} \\
\hat{\phi}_{N}
\end{array}\right)}_{\hat{\boldsymbol{u}}_{N}}=\underbrace{\left(\begin{array}{c}
\hat{F} \\
\hat{T}
\end{array}\right)}_{\hat{\boldsymbol{F}}_{N}} .
$$

\section{B. Modeling of the transducer}

The transducer, a spherical magnet that is fixed to one end of the beam, is modeled as a rigid sphere with mass $m_{M}$ and moment of inertia $I_{M}$. The equations of motion of the spherical magnet are set up in accordance with the freebody diagram shown in Fig. 3 and small angles of rotation $\phi_{N}$ are assumed:

$$
\begin{aligned}
m_{M} \ddot{w}_{M} & =m_{M}\left(\ddot{w}_{N}-a \ddot{\phi}_{N}\right)=Q_{M}, \\
I_{M} \ddot{\phi}_{M} & =I_{M} \ddot{\phi}_{N}=M_{M}+a Q_{M},
\end{aligned}
$$

where $a$ is the distance between the end of the tube and the center of mass of the sphere. Assuming all functions to be harmonic in time and suppressing the time dependence $e^{i \omega t}$ yields the dynamic-stiffness matrix:

$$
\hat{\mathbf{K}}_{M}=-\omega^{2}\left(\begin{array}{cc}
m_{M} & -a m_{M} \\
-a m_{M} & I_{M}+a^{2} m_{M}
\end{array}\right)
$$

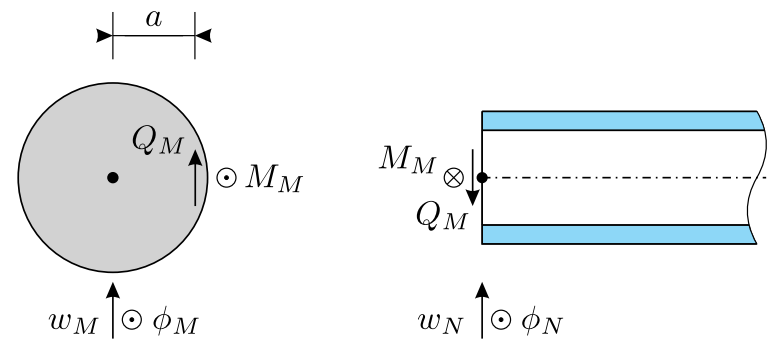

FIG. 3. Free-body diagram of the spherical magnet. The displacement and rotation of the center of mass of the spherical magnet are given by $w_{M}$ and $\phi_{M}$, respectively. The displacement and rotation of the tip of the beam are given by $w_{N}$ and $\phi_{N}$, which are equivalent to the nodal displacement and rotation. The shear force $Q_{M}$ acts at a distance $a$ from the center of mass of the spherical magnet.

\section{Computation of the excitation signal}

\section{Without reflections}

When computing the excitation moment for the transducer, we first consider the simplest case, where the wavelet undergoes no reflections between the focal point and the transducer. Here, the glass tube, which is of finite length in reality [Fig. 4(a)], is modeled as two semi-infinite spectral elements connected by a single node [Fig. 4(b)]. The bending moment in the left beam is

$$
\hat{M}_{L}(x)=-E I\left[i k_{1} \hat{A} e^{i k_{1}\left(x-x_{F}\right)}+i k_{2} \hat{B} e^{i k_{2}\left(x-x_{F}\right)}\right] .
$$

As we want the shape of the Ricker wavelet to be contained only in the first mode, we set $\hat{M}_{\text {Ricker }}=i k_{1} E I \hat{A}$ and obtain a value for $\hat{A}$. The value for $\hat{B}$ can be chosen arbitrarily. Thus, we can compute the shape of the dispersed Ricker wavelet $\hat{\Phi}$ at the transducer by evaluating the dynamic shape function $\hat{\Phi}=\hat{\phi}_{L}(0)$.

To obtain the excitation signal from the dispersed Ricker wavelet $\hat{\Phi}$, we first have to time reverse the wavelet by taking the complex conjugate [7] in the frequency domain $\hat{\Phi}^{*}$. Again, we want the wavelet to be contained in the first mode only and set $\hat{A}=\hat{\Phi}^{*}$. It remains to determine the moment $\hat{T}$ on the spherical magnet that would produce exactly this wavelet. To this end, we set up another spectral-element model consisting of one semiinfinite beam element connected to the spherical magnet [Fig. 4(c)]. Equating the loads at the (imaginary) joint yields the dynamic stiffness:

$$
\left(\hat{\mathbf{K}}_{R}+\hat{\mathbf{K}}_{M}\right)\left(\begin{array}{cc}
R_{1} & R_{2} \\
1 & 1
\end{array}\right)\left(\begin{array}{c}
\hat{A} \\
\hat{B}
\end{array}\right)=\left(\begin{array}{l}
0 \\
\hat{T}
\end{array}\right)
$$

where the external force $\hat{F}$ is set to zero because the transducer cannot produce an external force. Thus, $\hat{B}$ is determined such that the shear force of the second mode cancels 
(a)

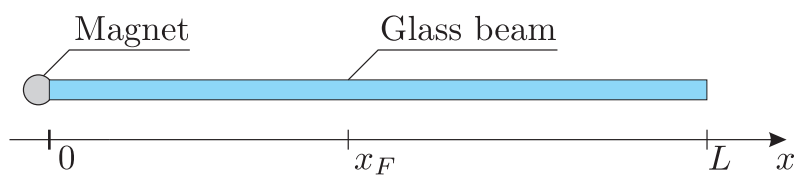

(b)

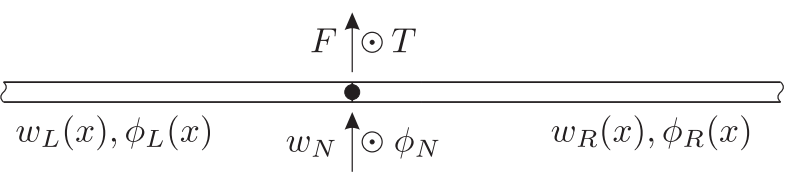

(c)

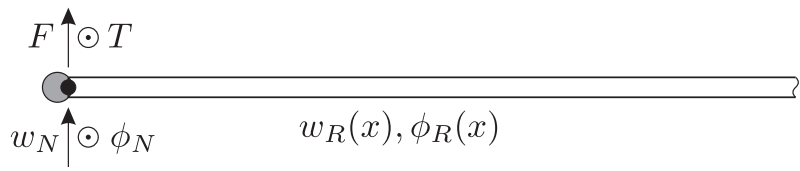

FIG. 4. Spectral-element models of the glass beam used for the computation of the excitation signal without reflections. (a) The glass beam with the position of the focal point $x_{F}$. (b) The spectral-element model used for the defocusing consists of two semi-infinite beams connected by a node at the focal point $x=$ $x_{F}$. The nodal displacement and nodal rotation are $w_{N}$ and $\phi_{N}$, respectively. The node is subjected to an external force $F$ and an external moment $T$. (c) The spectral-element model used for the computation of the excitation moment $T$ consists of one semi-infinite beam connected to a rigid sphere at $x=0$.

out the shear force of the first mode, and subsequently the excitation moment $\hat{T}$ is computed.

\section{With reflections}

For wavelets going through reflections, the simulation of the defocusing is extended. For every reflection, a separate semi-infinite beam element is used to simulate the propagation of the reflected wave away from the reflecting end of the beam. Separate elements are used to clearly distinguish between incident and reflected waves. To determine the shape of the reflected wave, we consider the free-body diagram [Fig. 5(a)] illustrating a reflection at the spherical magnet. Equating the loads at the tip of the beam yields

$$
\left(\begin{array}{c}
\hat{Q}_{M} \\
\hat{M}_{M}
\end{array}\right)+\left(\begin{array}{c}
\hat{Q}(0) \\
\hat{M}(0)
\end{array}\right)=\hat{\boldsymbol{F}}_{M}+\hat{\boldsymbol{F}}(0)=\mathbf{0}
$$

The loading $\hat{\boldsymbol{F}}(0)$ consists of a part produced by the incident wave $\hat{\boldsymbol{F}}_{\text {in }}$ and a part produced by the reflected wave $\hat{\boldsymbol{F}}_{\text {out }}$. Furthermore, the dynamic-stiffness matrix $\hat{\mathbf{K}}_{M}$ of the spherical magnet is used to express $\hat{\boldsymbol{F}}_{M}$ in terms of $\hat{\boldsymbol{u}}(0)=\hat{\boldsymbol{u}}_{\text {in }}+\hat{\boldsymbol{u}}_{\text {out }}$ :

$$
\begin{gathered}
\hat{\mathbf{K}}_{M}\left(\hat{\boldsymbol{u}}_{\text {in }}+\hat{\boldsymbol{u}}_{\text {out }}\right)+\hat{\boldsymbol{F}}_{\text {in }}+\hat{\boldsymbol{F}}_{\text {out }}=\mathbf{0}, \\
\hat{\boldsymbol{F}}_{\text {out }}=-\left(\mathbf{I}+\hat{\mathbf{K}}_{M} \hat{\mathbf{K}}_{R}^{-1}\right)^{-1}\left(\mathbf{I}-\hat{\mathbf{K}}_{M} \hat{\mathbf{K}}_{L}^{-1}\right) \hat{\boldsymbol{F}}_{\text {in }},
\end{gathered}
$$

(a)

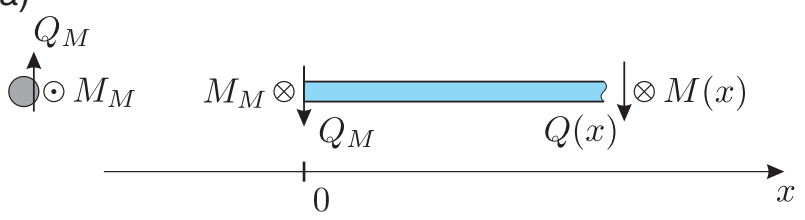

(b)

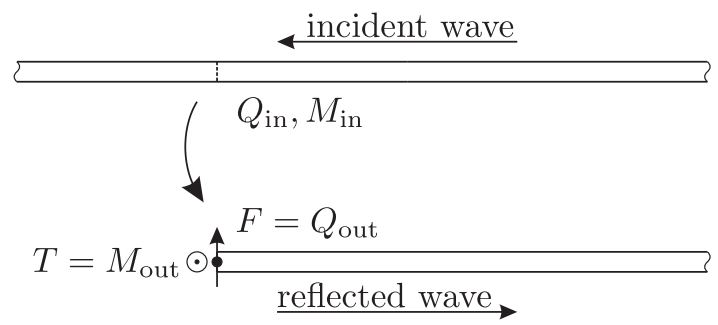

FIG. 5. Spectral-element model used to compute reflections. (a) Free-body diagram. (b) Spectral-element model emulating a reflection at the spherical magnet or a free end. The load of the reflected wave $\left(Q_{\text {out }}, M_{\text {out }}\right)$ is derived from the load of the incident wave $\left(Q_{\text {in }}, M_{\text {in }}\right)$. Subsequently, the propagation of the reflected wave is computed with a second semi-infinite beam element by setting the nodal load $F=Q_{\text {out }}$ and $T=M_{\text {out }}$.

where I is the identity matrix. Thus, the boundary condition $\hat{\boldsymbol{F}}_{\text {out }}$ for the simulation of the reflected wave can be computed in terms of the incident wave $\hat{\boldsymbol{F}}_{\text {in }}$ [Fig. 5(b)]. For reflections at the free end, the dynamic-stiffness matrix $\hat{\mathbf{K}}_{M}$ is set to zero, which corresponds to the zero-shearforce and zero-bending-moment condition. On the basis of the deflection of the reflected wave $\hat{\boldsymbol{u}}_{\text {out }}$, the shape of the dispersed and reflected Ricker wavelet at the transducer $\hat{\Phi}$ is determined and the excitation moment $\hat{T}$ is computed as explained above.

\section{Optimal signal composition}

Subsequently, the $n+1$ excitation signals for the individual wavelets, which focus at the focal point after zero to $n$ reflections, have to be summed up to one excitation signal that can be fed to the transducer. To do this in an optimal way, we introduce the amplification factor $A_{r}=m_{r} / T_{r}$. This is the ratio of the maximum bending moment $m_{r}$ produced by a wavelet $W_{r}$ at the focal point to the maximum moment $T_{r}$ required at the transducer [Fig. 6(a)], which is also proportional to the current flowing through the coil. In other words, the amplification factor $A_{r}$ quantifies the efficiency of the wavelet $W_{r}$.

A typical measurement of the amplification factor is shown in Fig. 6(b). It is obtained by our measuring the maximum bending moment at the focal point produced by 31 distinct wavelets having undergone up to 30 reflections. For small numbers of reflections $r$, the amplification is close to 1 , because the covered distance between the transducer and the focal point is still small and the effect 
(a)

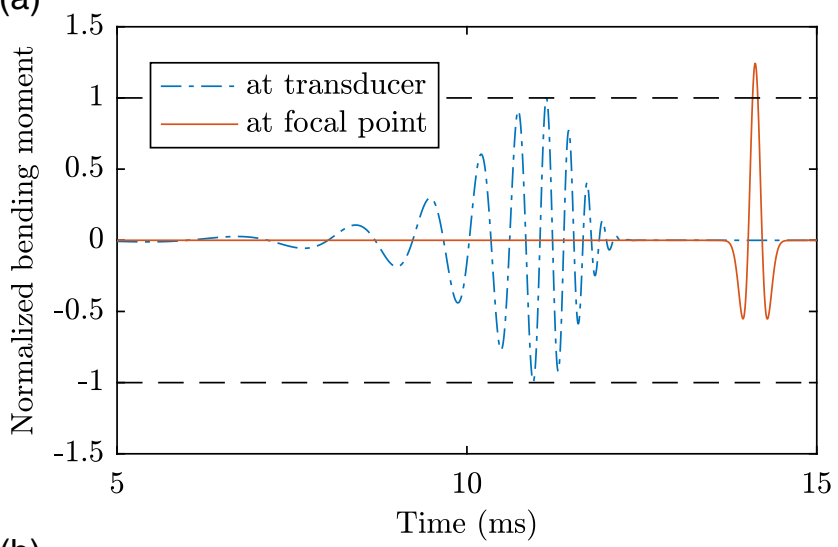

(b)

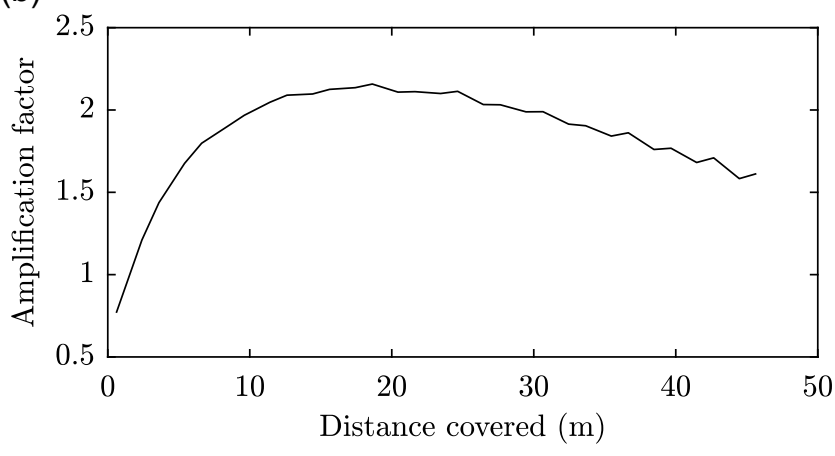

FIG. 6. Definition and measurement of the amplification factor. (a) Time history of the normalized bending moment of the same wavelet at the transducer (dot-dashed line) and at the focal point (solid line). The time history of the bending moment at the transducer consists of the propagating mode and the evanescent mode. At the focal point, only the propagating mode is present. The amplification factor is the ratio of the maximum bending moment at the focal point and the maximum bending moment at the transducer (here 1.25). (b) Measurement of the amplification factor for increasing distances between the focal point and the transducer.

of dispersion is not strong. The amplification increases for larger $r$, as the distance covered by the wave increases and the dispersion spreads the frequency content more and more. For large distances, however, the energy loss through damping becomes dominant and the amplification factor decreases and goes to zero.

To summarize, we obtain $n+1$ excitation signals for wavelets, which will each produce a maximum bending moment $m_{r}$ at the focal point. The final excitation signal is the weighted sum of all $n+1$ signals and is delimited by the maximum current provided by the current source. Likewise, the resulting maximum bending moment at the focal point is the weighted sum of all $n+1$ bending moments $m_{r}$ and shall be maximized. This corresponds to the problem statement of linear programming, for which an optimal solution can be found with use of the simplex algorithm, for example.

\section{EXPERIMENTAL RESULTS}

The wave-focusing method is demonstrated in experiments, where the strong bending-moment pulse at the focal point leads to dynamic fracture of the glass beam. After presentation of the experimental setup, the mensuration of the glass beam and the transducer is discussed. Finally, the results of the wave-focusing experiments are illustrated.

\section{A. Experimental setup}

In the experiments, a 1.5 -m-long glass tube with circular cross section is used as a waveguide (for more details see Table I). The surface of the glass tube is slightly roughened at multiple locations with use of grade- 60 sandpaper to decrease its bending strength [Fig. 7(a)]. The transducer consists of a spherical magnet (diameter $6 \mathrm{~mm}$ ), which is glued to one end of the glass tube, with the polarity of the magnet perpendicular to the axis of the tube. The spherical magnet is then inserted into an electromagnetic coil, which is aligned with the axis of the tube [Fig. 7(b)]. This constellation of the magnetic fields of the magnet and the electromagnetic coil results in a moment acting on the magnet that is perpendicular to the polarity of the magnet and the axis of the beam [32]. Thus, bending waves are excited at the end of the beam with the displacement lying in the plane perpendicular to the moment. The electromagnetic coil is driven with a voltage-controlled current source because the electromagnetic field produced by the coil and the resulting moment on the magnet are proportional to the current flowing through the coil. The glass beam is placed horizontally on several foam supports to reduce disturbances resulting from the mounting. The lateral displacement of the beam is measured with a laser Doppler vibrometer (Polytec OFV 303 sensor head, VD-02 velocity decoder), which can be moved in the axial direction to observe the wave propagation. For this purpose, the beam is fitted with reflective tape at several locations.

\section{B. Pulse shape}

For the shape of the focused pulse, the Ricker wavelet (also known as a "Mexican hat wavelet") is chosen because it has zero mean and a well-defined frequency content. The pulse width, and thereby the frequency content, is varied in multiple experiments. For the results presented here, a pulse width of $\sigma=0.1 \mathrm{~ms}$ is used, which corresponds to a center frequency of $2.5 \mathrm{kHz}$ (see Fig. 2).

\section{Phase velocity}

For the energy focusing to work, the frequencydependent phase velocity of the propagating wave mode has to be characterized with high precision. Therefore, the phase velocity is measured for every beam by excitation of the transducer with a chirp and measurement of the resulting wavelet at different positions along the beam. The 
TABLE I. Material data and geometry of the experimental setup.

\begin{tabular}{ll}
\hline \hline Glass beam & \\
Type & Schott AR-Glas ${ }^{\circledR}$ \\
Length & $1.5 \mathrm{~m}$ \\
Outside diameter & $4 \mathrm{~mm}$ \\
Wall thickness & $0.5 \mathrm{~mm}$ \\
Magnet & \\
Type & Neodym N38 (Webcraft \\
& AG, Uster, \\
& Switzerland) \\
Diameter & $6 \mathrm{~mm}$ \\
Mass & $0.86 \mathrm{~g}$ \\
Residual magnetism & $1.22-1.26 \mathrm{~T}$ \\
Electromagnetic coil & \\
Inside diameter & $7 \mathrm{~mm}$ \\
Length & $9 \mathrm{~mm}$ \\
Number of turns & 90 \\
Wire diameter & $0.5 \mathrm{~mm}$ \\
Material & Copper \\
Power amplifier & \\
Type & PA401 (Rohrer GmbH, \\
& Munich, Germany) \\
Maximum current & $\pm 10 \mathrm{~A}$ \\
Maximum voltage & $\pm 40 \mathrm{~V}$ \\
\hline \hline
\end{tabular}

comparison of the phase of the measured wavelets yields the phase velocity. Subsequently, the phase velocity curve obtained with Timoshenko beam theory is fitted to the experimental data by adjustment of the Young's modulus $E$ and the shear modulus $G$ (Fig. 8). These two parameters, together with the geometry of the beam, suffice to describe the phase velocity of the first bending wave mode of the glass beam.
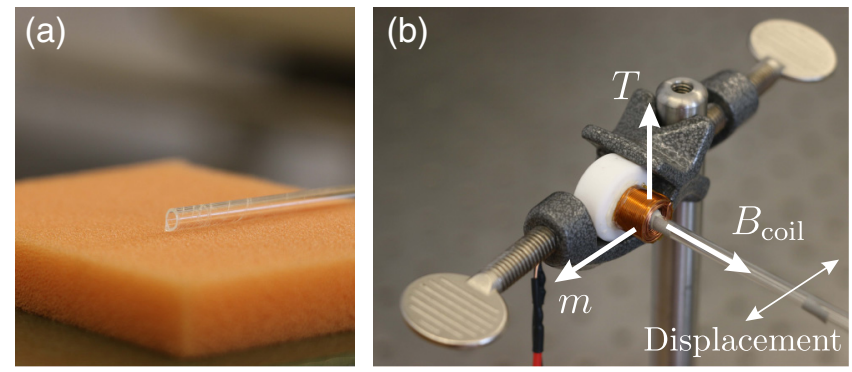

FIG. 7. Experimental setup. (a) Glass tube that has been fractured by wave focusing. The surface of the glass tube has been slightly roughened with sandpaper to reduce the breaking load. (b) The electromechanical transducer consists of a spherical magnet with magnetic moment $m$, which is glued to one end of the glass beam. An external moment $T$ is generated on the spherical magnet by its placement in the magnetic field $B_{\text {coil }}$ of an electromagnetic coil.

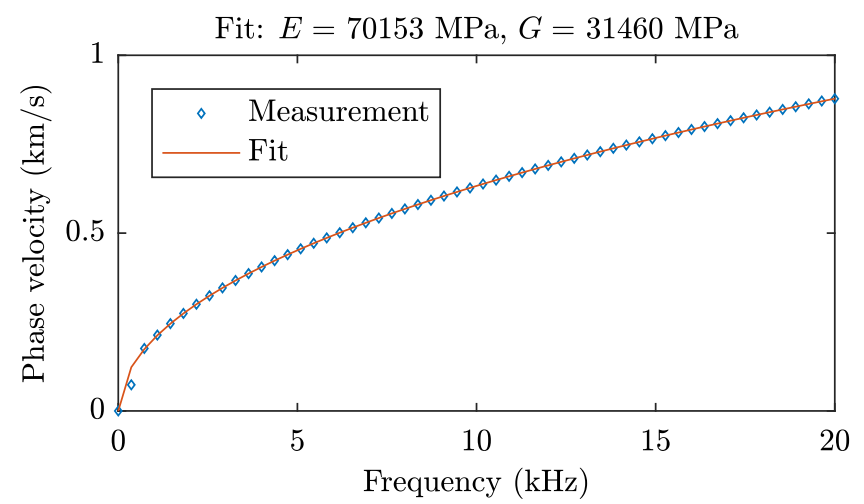

FIG. 8. Measured phase velocity of the flexural waves and phase velocity according to Timoshenko beam theory after fitting of the Young's modulus $E$ and shear modulus $G$.

\section{Measurement of the bending moment}

To assess the wave-focusing procedure, the shape and amplitude of the bending-moment pulse at the focal point have to be determined. As the bending moment cannot be measured directly, it is derived from the measurement of the lateral surface velocity obtained with the laser Doppler vibrometer as follows.

As long as we are far enough from the ends of the beam, the deformation of the beam is governed only by the propagating wave mode, because the evanescent wave mode has decayed substantially. Thus, the deflection of the beam can be written as the superposition of a left-traveling wave and a right-traveling wave:

$$
\begin{aligned}
& \hat{w}(x)=R_{1} \hat{A} e^{-i k_{1} x}-R_{1} \hat{C} e^{i k_{1}(x-L)}, \\
& \hat{\phi}(x)=\hat{A} e^{-i k_{1} x}+\hat{C} e^{i k_{1}(x-L)} .
\end{aligned}
$$

Accordingly, the velocity normal to the surface and the bending moment are

$$
\begin{array}{r}
\hat{v}(x)=i \omega\left[R_{1} \hat{A} e^{-i k_{1} x}-R_{1} \hat{C} e^{i k_{1}(x-L)}\right], \\
\hat{M}(x)=-E I\left[-i k_{1} \hat{A} e^{-i k_{1} x}+i k_{1} \hat{C} e^{i k_{1}(x-L)}\right],
\end{array}
$$

which can be rewritten as

$$
\hat{M}(x)=\frac{E I k_{1}}{R_{1} \omega} \hat{v}(x) .
$$

\section{E. Measurement of the transducer strength}

To measure the amplification of the bending moment experimentally, both the bending moment at the focal point and the moment produced by the transducer have to be determined. The bending moment at the focal point can be derived from the lateral surface velocity as shown earlier [Eq. (16)]. However, this cannot be done for the 


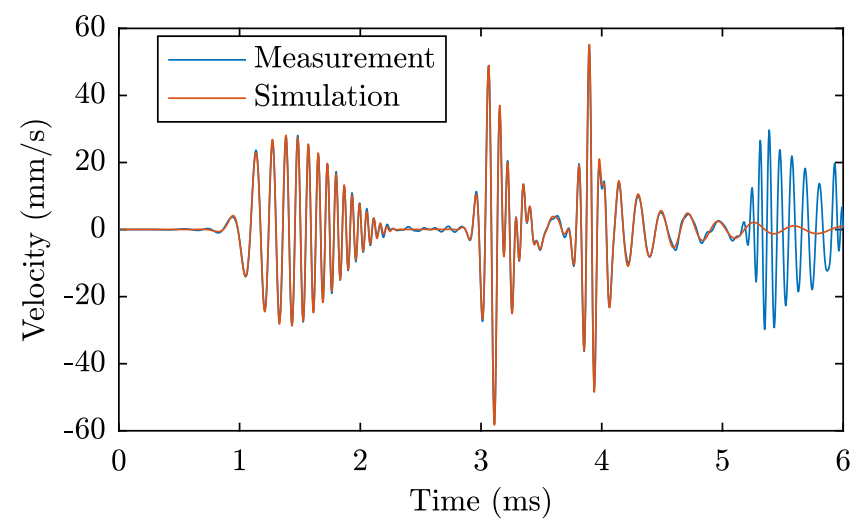

FIG. 9. Comparison of measured and simulated lateral surface velocity of the beam $v(x=0.5 \mathrm{~m}, t)$ at a distance of $0.5 \mathrm{~m}$ from the transducer. The time history shows the arrival of the excitation chirp between 1 and $2 \mathrm{~ms}$. The first and second reflections arrive at around 3 and $4 \mathrm{~ms}$, respectively. Further reflections are not accounted for in the simulation. The strength of the transducer is determined by scaling of the simulated time history to the measured one.

moment produced by the transducer, as the electromagnetic coil obstructs the laser. Moreover, the evanescent mode is present close to the transducer, so the derivations presented before are not valid.

Instead, the strength of the transducer is determined by measuring the lateral surface velocity close to the transducer and deducing the moment acting on the magnet with a simulation. The same excitation signal is applied as the current in the experiment and as the external moment in the simulation. Subsequently, the measured and simulated lateral surface velocities at a certain point along the beam are compared. They differ only in a scaling factor (Fig. 9), because the moment on the spherical magnet is proportional to the magnetic field inside the coil, which is again proportional to the current through the coil. This scaling factor corresponds to the strength of the transducer in newton meters per ampere.

\section{F. Measurement of the beam length}

Aside from the phase velocity, the beam length is an important quantity that has to be measured to achieve precise wave focusing. If the length of the beam in the simulation does not match the exact length of the physical beam, the individual wavelets will not focus at the same location, which may lead to destructive interference.

To determine the length of the beam, a procedure similar to that for the measurement of the transducer strength is chosen. Again, the measured and simulated lateral surface velocities at a certain point along the beam are compared. Subsequently, the length of the beam is adapted iteratively in the simulation such that the arrival times of the

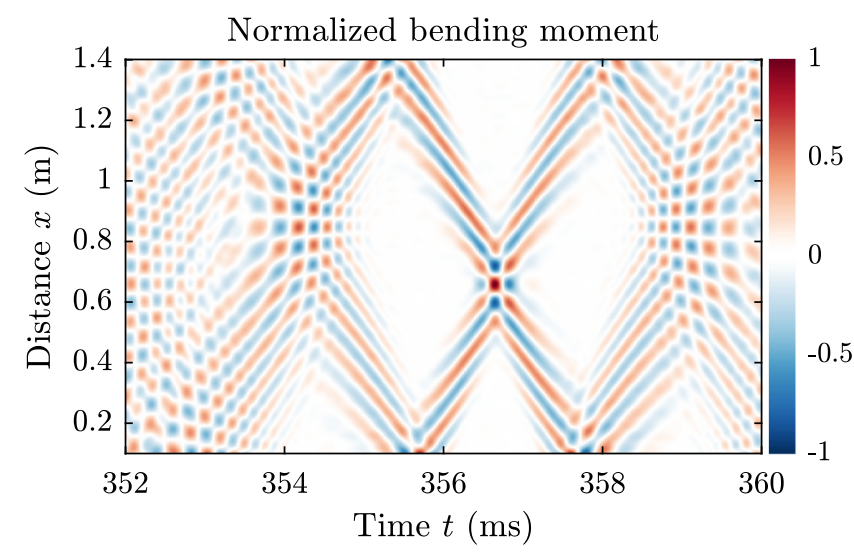

FIG. 10. Time-space map of the measured bending moment (normalized). The bending waves are produced by a transducer fixed at one end of the beam $(x=0 \mathrm{~m})$ and focus at $x=0.65 \mathrm{~m}$, $t=356.7 \mathrm{~ms}$. The excitation starts at $t=0 \mathrm{~ms}$ and ends at $t=356 \mathrm{~ms}$. The earliest wavelet undergoes 30 reflections before reaching the focal point.

direct wavelet, the first reflection, and the second reflection coincide in the simulation and the experiment. A typical comparison between simulated and measured lateral surface velocities is presented in Fig. 9, showing that very close agreement between the two can be achieved.

\section{G. Results}

In a set of experiments, a time-space map of the wave field inside the beam is constructed to observe the wave focusing. For these experiments, the entire length of the beam is fitted with reflective tape necessary for the laser Doppler vibrometer measurements and the beam is not sanded, so it would not break. The mensuration of the beam and the computation of the excitation signal are done as explained earlier. Subsequently, the beam is excited with the resulting excitation signal repetitively, and the lateral

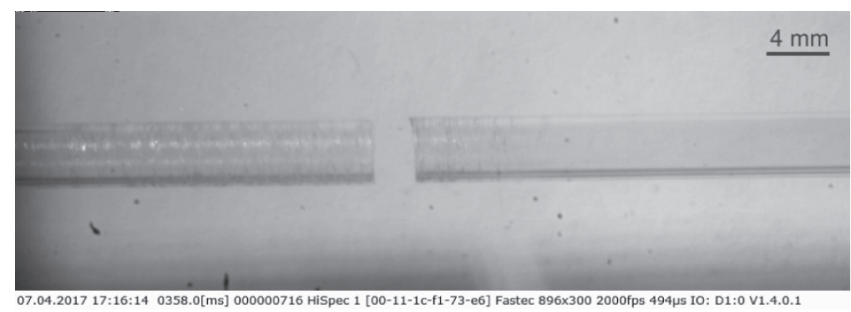

VIDEO 1. The fracturing of the glass beam (recording 2000 frames/s, replay 24 frames/s). The recording starts with the excitation of the beam $(t=0 \mathrm{~ms})$, whereas the replay starts at $t=276$ $\mathrm{ms}$. The beam breaks at $t=349 \mathrm{~ms}$. After breaking, the righthand side of the beam starts to move because it is supported by only one foam cushion and loses balance. The surface of the beam was sanded only in the vicinity of the focal point. In later experiments, larger areas of the beam were sanded. 


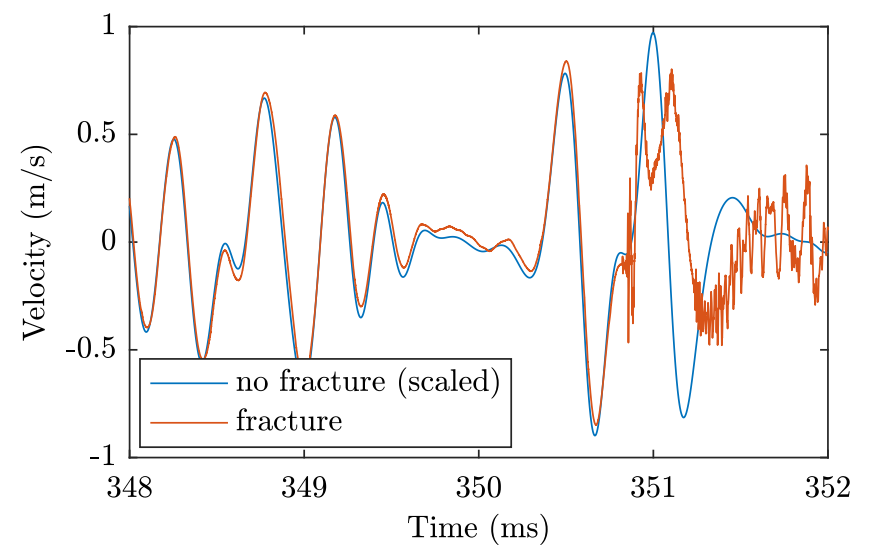

FIG. 11. Measurement of the lateral surface velocity at a distance $x=0.5 \mathrm{~m}$ from the transducer, while the waves focus at $x=0.65 \mathrm{~m}$. For the blue curve, only $40 \%$ of the available power is used so as not to break the beam. Subsequently, the blue curve is scaled up by 2.5 to match the red curve, for which full power is applied to break the beam. Both curves match very well before the beam breaks at approximately $t=351 \mathrm{~ms}$ and differ considerably afterwards. Furthermore, waves that have much higher frequency content and result from the energy released during the fracture (acoustic emission) can be seen in the red curve.

surface velocity is measured at $1-\mathrm{mm}$ intervals along the beam. The resulting time-space map of the bending moment is depicted in Fig. 10. It shows how the bending waves, which are excited at $x=0 \mathrm{~m}$ between $t=0 \mathrm{~ms}$ and $t=357 \mathrm{~ms}$, focus at $x=0.65 \mathrm{~m}$ and produce a strong bending-moment pulse.

In experiments with beams that had not been sanded, a maximum bending moment of $184 \times 10^{-3} \mathrm{Nm}$ is measured at the focal point when 30 reflections are taken into account. This is more than 20 times larger than the maximum bending moment the transducer is capable of producing (around $8.3 \times 10^{-3} \mathrm{Nm}$ ). After the beams are sanded and thereby their bending strength reduced to about $117 \times 10^{-3} \mathrm{~N} \mathrm{~m}$ (measured in quasistatic four-point bending tests), we are able to induce dynamic fracture at the focal point as can be seen in Video 1 and Fig. 11.

\section{DISCUSSION AND CONCLUSION}

In summary, we present a method to create a short, highpower bending-wave pulse in a beam at a position away from the transducer. The excitation signal is computed in a time reversed spectral-element simulation and consists of 31 wavelets, for which up to 30 reflections are taken into account. The 31 wavelets are composed with linearprogramming algorithms to obtain an optimal excitation signal. Thus, a bending-moment pulse with a maximum amplitude of $184 \times 10^{-3} \mathrm{~N} \mathrm{~m}$ is achieved in experiments, where a transducer capable of producing only $8.3 \times 10^{-3}$ $\mathrm{Nm}$ is used. This corresponds to an amplification of the bending moment by a factor of more than 20 by wave focusing. Moreover, the amplitude of the bending-moment pulse proves to be large enough to induce dynamic fracture of the glass beam.

The position of the focal point can be set with very high accuracy as both the phase velocity of the flexural waves and the length of the beam are determined precisely. Larger inaccuracies in the measurement of these two quantities would lead not to imprecision in the position of the focal point but rather to a complete breakdown of the focusing method. This is because the individual wave packages would focus at different locations and at different instances of time, possibly leading to destructive interference.

The location of fracture, on the other hand, is dictated by the critical bending moment required to break the beam, and the amplitude and width of the bending-moment pulse. Moreover, the critical bending moment is not constant along the beam, due to the sanding. Hence, we can only guarantee the location of fracture to be inside a certain perimeter of the focal point, which is governed by the wavelength chosen. In the experiments, the beams broke within a distance of $\pm 5 \mathrm{~mm}$ from the focal point, where the bending moment is still $95 \%$ of its peak value.

For all experiments presented here, the same pulse width with a center frequency of $2.5 \mathrm{kHz}$ is used. However, it is worth mentioning that the wave-focusing method works in the complete frequency domain below the cutoff frequency of the second wave mode. The accuracy of the location of fracture could be increased by going to higher frequencies, thereby reducing the wavelengths and the size of the focal spot. Yet, dispersion is less pronounced at higher frequencies (see Fig. 8), and the transducer bandwidth and material damping may add further difficulties. By increase of the number of reflections, the amplification could be increased even further, but the gain due to additional reflections decreases, as more energy is lost through material damping. On the other hand, the number of reflections required to achieve dynamic fracture could be reduced by use of a stronger electromechanical transducer (e.g., by use of a more powerful current source).

By precisely modeling the propagation of flexural waves in a glass beam, we design and create a bending-moment pulse at an arbitrary location away from the actuator. With the same method, other quantities, such as shear force, deflection, or surface velocity, could also be tailored. What is more, the wave-focusing technique could be transferred to other dispersive waves (e.g., water waves, or light in dispersive media) to focus energy.

Applying the wave-focusing technique to induce dynamic fracture at an arbitrary location might be interesting for high-strain-rate bending tests in fracture mechanics. By setting the shape of the pulse, one is able to directly control the strain rate at the location of fracture. Moreover, no external loads are applied during the fracturing process. This eliminates the stress concentrations that typically occur in the vicinity of the indenter and the support in 
classic mechanical tests, such as three- and four-point bending [33].

\section{ACKNOWLEDGMENTS}

We thank Robert Ernst, Stefan Künzli, and Martin Veidt for getting this project started and all the work they put into it. We thank Mario Weder for providing help with MATLAB, LABVIEW, and the experimental setup. This work was entirely funded by ETH Zurich.

[1] K. F. Graff, Wave Motion in Elastic Solids (Clarendon Press, Oxford, 1975).

[2] P. Wilcox, M. Lowe, and P. Cawley, The effect of dispersion on long-range inspection using ultrasonic guided waves, NDT\&E Int. 34, 1 (2001).

[3] R. Ernst and J. Dual, Acoustic emission localization in beams based on time reversed dispersion, Ultrasonics 54, 1522 (2014).

[4] R. Ernst and J. Dual, Quantitative guided wave testing by applying the time reversal principle on dispersive waves in beams, Wave Motion 58, 259 (2015).

[5] R. Ernst, F. Zwimpfer, and J. Dual, One sensor acoustic emission localization in plates, Ultrasonics 64, 139 (2016).

[6] T. Leutenegger and J. Dual, Detection of defects in cylindrical structures using a time reverse method and a finitedifference approach, Ultrasonics 40, 721 (2002).

[7] M. Fink, Time reversed acoustics, Phys. Today 50, 34 (1997).

[8] R. K. Ing and M. Fink, Self-focusing and time recompression of Lamb waves using a time reversal mirror, Ultrasonics 36, 179 (1998).

[9] J. de Rosny and M. Fink, Publisher's Note: Overcoming the Diffraction Limit in Wave Physics using a Time-reversal Mirror and a Novel Acoustic Sink [Phys. Rev. Lett. 89, 124301 (2002)], Phys. Rev. Lett. 89, 219901(E) (2002).

[10] S. Yon, M. Tanter, and M. Fink, Sound focusing in rooms: The time-reversal approach, J. Acoust. Soc. Am. 113, 1533 (2003).

[11] G. Lerosey, J. de Rosny, A. Tourin, A. Derode, G. Montaldo, and M. Fink, Time Reversal of Electromagnetic Waves, Phys. Rev. Lett. 92, 1939041 (2004).

[12] G. Lerosey, J. de Rosny, A. Tourin, and M. Fink, Focusing beyond the diffraction limit with far-field time reversal, Science 315, 1120 (2007).

[13] F. Lemoult, G. Lerosey, J. de Rosny, and M. Fink, Resonant Metalenses for Breaking the Diffraction Barrier, Phys. Rev. Lett. 104, 203901 (2010).

[14] F. Lemoult, M. Fink, and G. Lerosey, Acoustic Resonators for Far-field Control of Sound on a Subwavelength Scale, Phys. Rev. Lett. 107, 064301 (2011).

[15] F. Lemoult, M. Fink, and G. Lerosey, A polychromatic approach to far-field superlensing at visible wavelengths, Nat. Commun. 3, 889 (2012).
[16] H. Kolsky, An investigation of the mechanical properties of materials at very high rates of loading, Proc. Phys. Soc. B 62, 676 (1949).

[17] W. Chen and B. Song, Split Hopkinson (Kolsky) Bar, Mechanical Engineering Series (Springer US, Boston, MA, 2011).

[18] R. J. Christensen, S. R. Swanson, and W. S. Brown, SplitHopkinson-bar Tests on Rock under Confining Pressure, Exp. Mech. 12, 508 (1972).

[19] S. Nemat-Nasser, J. B. Isaacs, and J. E. Starrett, Hopkinson techniques for dynamic recovery experiments, Proc. R. Soc. Lond. A 435, 371 (1991).

[20] J. Harding, E. O. Wood, and J. D. Campbell, Tensile testing of materials at impact rates of strain, J. Mech. Eng. Sci. 2, 88 (1960).

[21] C. C. Roth, G. Gary, and D. Mohr, Compact SHPB system for intermediate and high strain rate plasticity and fracture testing of sheet metal, Exp. Mech. 55, 1803 (2015).

[22] W. E. Baker and C. H. Yew, Strain-rate effects in the propagation of torsional plastic waves, J. Appl. Mech. 33, 917 (1966).

[23] K. Tanaka and K. Toshihide, Impact bending test on steel at low temperatures using a Split Hopkinson Bar, Bull. JSME 23, 1736 (1980).

[24] T. Weerasooriya, P. Moy, D. T. Casem, M. Cheng, and W. Chen, A four-point bend technique to determine dynamic fracture toughness of ceramics, J. Am. Ceram. Soc. 89, 990 (2006).

[25] D. T. Casem, A. K. Dwivedi, J. J. Swab, J. C. Wright, and A. B. Mondal, Analysis of a Three-Bar Kolsky apparatus for high-rate three-point flexure, J. Dynamic Behavior Mater. 1, 75 (2015).

[26] S. Henschel and L. Krüger, Dynamic crack initiation measurements in a four-point split Hopkinson bending device, Eng. Fract. Mech. 133, 62 (2015).

[27] W. Böhme and J. F. Kalthoff, The behavior of notched bend specimens in impact testing, Int. J. Fract. 20, R139 (1982).

[28] P. Forquin, Brittle materials at high-loading rates: An open area of research, Philos. Trans. R. Soc. A: Math. Phys. Eng. Sci. 375, 20160436 (2017).

[29] A. M. Weiner, Ultrafast optical pulse shaping: A tutorial review, Opt. Commun. 284, 3669 (2011).

[30] S. P. Timoshenko, LXVI. On the correction for shear of the differential equation for transverse vibrations of prismatic bars, Philos. Mag. 41, 744 (1921).

[31] J. F. Doyle, Wave Propagation in Structures: Spectral Analysis using Fast Discrete Fourier Transforms (SpringerVerlag, New York, 1997).

[32] B. D. Cullity and C. D. Graham, Introduction to Magnetic Materials (John Wiley \& Sons, Inc., Hoboken, NJ, 2008), $2^{\text {nd }}$ edition.

[33] W. C. Cui and M. R. Wisnom, Contact finite element analysis of three- and four-point short-beam bending of unidirectional composites, Compos. Sci. Technol. 45, 323 (1992). 